\title{
In Situ Detection and Identification of Microorganisms at Single Colony Resolution Using Spectral Imaging Technique
}

\author{
Kanae Miyazawa ${ }^{1}$, Ken-ichi Kobayashi $^{1}$, Shigeki Nakauchi ${ }^{1}$, and Akira Hiraishi ${ }^{2}$ \\ ${ }^{1}$ Department of Information and Computer Sciences, \\ Toyohashi University of Technology, \\ 1-1 Hibarigaoka, Tempaku, Toyohashi, Aichi 441-8580, Japan \\ \{kanae, kobayashi04, naka\}@bpel.ics.tut.ac.jp \\ http: //www.bpel.ics.tut.ac.jp/ \\ ${ }^{2}$ Department of Ecological Engineering, \\ Toyohashi University of Technology, \\ 1-1 Hibarigaoka, Tempaku, Toyohashi, Aichi 441-8580, Japan \\ hiraishi@eco.tut.ac.jp
}

\begin{abstract}
In situ detection and identification of microorganisms in the environment are important in general microbial ecology. Also the rapid inspection of microbial contamination at food processing plant is urgent task. We propose a method of detecting and identifying microorganisms for rapid inspection using spectral imaging technique. Spectral images of photosynthetic and nonphotosynthetic bacterial colonies having different absorption spectra in near infrared wavelength region were measured directly from Petri dish. Bacterial region in the images was first detected and then identified using multiple discriminant analysis. Detection and identification errors for various sized colonies were analyzed. As the result, colonies with diameters of 100 and $300 \mu \mathrm{m}$ were detected and identified with sufficient accuracy, respectively. This means the time for detection and identification can be shorten less than a half and about several weeks compared with the conventional methods.
\end{abstract}

\section{Introduction}

In situ detection and identification of microorganisms in the environment are important in general microbial ecology. Also food poisoning incidents caused by microorganisms have frequently occurred recently and the inspection at food processing plant is conducted with scrupulous care. Traditional culturing techniques, in which microorganisms are cultured for a few days and inspected by human eyes, is still widely used for detecting microbial contamination in food products. However, it takes relatively long time and the whole plant is possibly contaminated before microorganisms are detected. In the worst case, the contaminated food products must be recalled. It makes not only huge economic loss but also compromises their reputation. Therefore, rapid inspection of microbial contamination at food processing plant is urgent task.[1]

Fluorescence microscopy, flow cytometry, polymerase chain reaction techniques have recently been the focus of attention for identification of microorganisms.[2],[3] These methods can be used as powerful tools for precise identification in laboratory. 
However, they are not appropriate ways for rapid food inspection at plants, because chemical pretreatment including poisonous staining is needed and/or the equipments are extremely expensive. Not precise identification of microorganisms but rapid screening is needed at the plants. Rapid inspection technique using shape information of growing microorganisms has been proposed and the equipment has come onto the market. This can be useful for rapid detection for relatively small size microorganisms called microcolony, however alien substances are possibly mis-detected as microorganisms. In the point of view, shape information is not enough for detection.

On the other hand, it is known that microorganisms like bacteria have typical absorption spectra at ultra violet (UV) and infrared (IR) wavelengths regions caused by pigment, protein and so on. In laboratory, spectrophotometer is usually used for identification of microorganisms. Sugiura et al.[4] proposed a simple method for absorption spectrophotometry to identify photosynthetic pigments of microbial mats using a portable spectrophotometer in the field. This method is simple and easy to use in the field. However, a large quantity of samples like mats are required for measurement, because the sample is placed between two slide glasses and the transmitted light is measured by putting samples between light source and detector. In this case, average spectra of microbial mats can be measured and it is not applied to food inspection which needs to detect and identify a single colony. To detect a single colony of target microorganisms from contaminated foods, imaging technique is necessary.

Spectral imaging technique, in which each pixel in an image has spectral information, has received a great deal of attention recently. Spectral imaging devices are under a rapid development and the applications of spectral imaging including UV and IR wavelengths regions are expected. Spectral imaging technique has been used to detect stained[5],[6] and unstained microbial colonies[7],[8] which are fully developed. In this study, we propose a method to detect and identify a single colony of live unstained microorganisms for rapid inspection using spectral imaging technique. The method was applied to various sized colonies and we investigated the limitation of the method.

The paper is organized as follows. In Section 2, we describe materials and methods consisted of sample bacteria used in the experiment, an experimental setup for spectral imaging, method for detection and identification of microbial colonies and evaluation method. The experimental results are shown in Section 3 and Section 4 gives the discussion and conclusions.

\section{Materials and Methods}

\subsection{Bacteria}

(a)Paracoccus pantotrophus JCM6892, (b)Rhodopseudomonas palustris DSM123, (c)Rhodobium marinum DSM2698 and (d)Rhodobacter veldkampii ATCC35703 were used as model organisms. Examples of bacterial colonies used in the experiment are shown in Figure 1(A), (B). The photos were taken by an RGB digital camera attached to an optical microscope with (A) 4- and (B) 10-power object lens, respectively. Both in (A) and (B), (b), (c) and (d) are photosynthetic bacteria having distinctive spectral absorption peaks caused by pigment-protein complexes with bacteriochlorophyll $a$ (Bchl $a$ ). A non-photosynthetic bacterium (a) which has no Bchl $a$ was also used as a 
control bacterium. These bacteria were cultured aerobically on agar plates under incandescent illumination and were used for experiment without any chemical pretreatment such as staining. The sizes of the colonies were about (A) 1-2 millimeter and (B) a few hundreds micrometer. Detection and identification of colonies in (A) may not difficult task for experts. However, colonies in (B) are difficult to detect because of its small size and almost impossible to identify even for experts because colonies are too thin and the colors are all reddish and transparent. Shape information cannot help us because all of them are flat and round. Figure 2 shows absorption spectra of bacterial cells measured with a conventional spectrophotometer. Distinctive peaks caused by pigment-protein complexes with Bchl $a$ can be seen in (b), (c) and (d), where (a) has no peaks at infrared region.

(A)

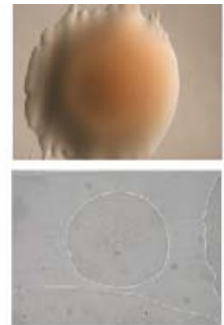

(a)

Paracoccus pantotrophus

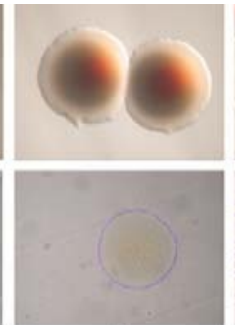

(b)

Rhodopseudomonas palustris

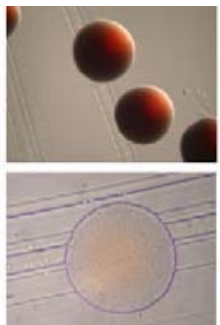

(c)

Rhodobium marinum

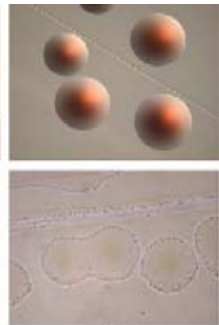

(d)

Rhodobacter veldkampii

Fig. 1. Four species of bacteria used in the experiment. (A) Relatively large size colonies taken with 4-power object lens, (B) microcolonies taken with 10-power object lens. The sizes of the colonies were about (A) 1-2 millimeter and (B) a few hundreds micrometer. (b)-(d) are photosynthetic bacteria having distinctive spectral absorption peaks caused by pigment-protein complexes with bacteriochlorophyll $a(\mathrm{Bchl} a)$ and (a) is a non-photosynthetic bacterium having no Bchl $a$
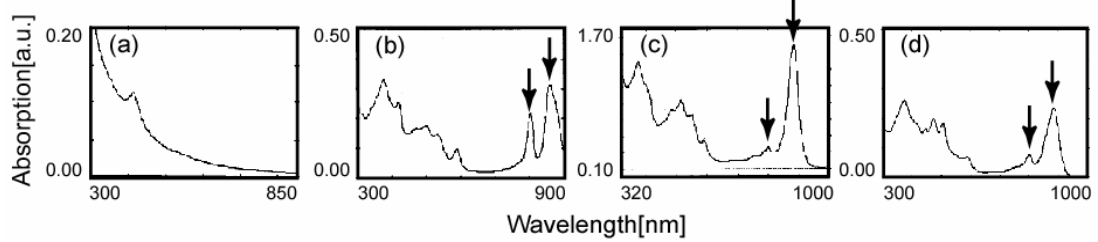

Fig. 2. Absorption spectra of bacterial cells measured with a conventional spectrophotometer. Distinctive peaks caused by pigment-protein complexes with Bchl $a$ can be seen at infrared region in (b), (c) and (d) as pointed by arrows, where (a) has no peaks at infrared region

\subsection{Experimental Setup}

An experimental setup for a spectral imaging system is shown in Figure 3. A Petri dish, whose cover was opened, was set on an optical microscope with a halogen lamp (Nikon SMZ-10 with 4-power zoom object lens, Nikon LABOPHOT-2 with 10power object lens). An intensity image of the bacterial colonies trans-illuminated by 
the halogen lamp was taken with a monochrome CCD camera (HAMAMATSU, ORCA-ER-1394) through an acousto-optic tunable filter (AOTF) (Brimrose, CVA100-0.4-1.0) whose transmitting wavelength can be electrically changed visible to near infrared region (400 to $1000 \mathrm{~nm}$ ). Wavelength range for taking images in this experiment was between 750 and $946 \mathrm{~nm}$ at $2 \mathrm{~nm}$ intervals, because photosynthetic bacteria could be identified with the spectral resolutions of $2 \mathrm{~nm}$ in the region. According to Lambert-Beer law, absorption spectral images were calculated using spectral images of colonies and reference. As the reference, a spectral image of a Petri dish with culture media, which was not included colonies, was used for each species. Reference images varied depending on the media whose ingredients were different, but not on the places where taking images. The experiment was done in laboratory environment with the temperature of $24-25$ degree Celsius.

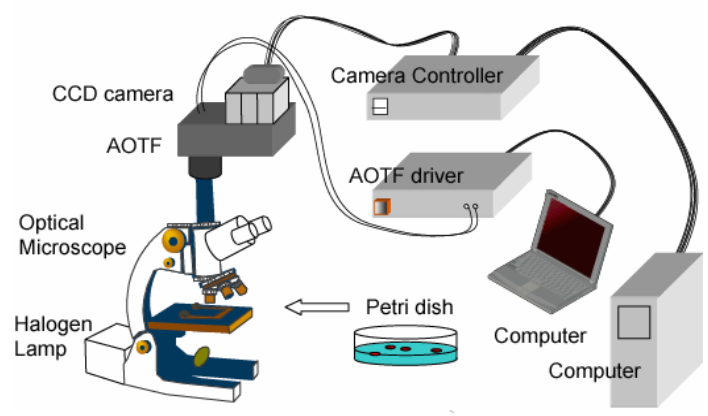

Fig. 3. Spectral imaging system. An intensity image of the bacterial colonies trans-illuminated by the halogen lamp was taken with a monochrome CCD camera through an acousto-optic tunable filter

\subsection{Detection and Identification}

Analysis consisted two phases, detection and identification. First, pixelwise detection was done by separating colony area and background area by multiple discriminant analysis (MDA) using mean absorption and standard deviation of the spectra. The mean absorption of colony area is theoretically high compared with that of background area which was consisted of Petri dish and culture media. Experimentally, however, the mean absorption of colony area is sometimes lower than that of background area due to the experimental artifacts. On the other hand, standard deviation of the spectra of colony area is high in infrared region because of absorption peaks caused by pigment-protein complexes with Bchl $a$. Therefore both mean absorption and standard deviation of the spectra were used for the detection in the first phase.

Next, pixelwise identification was done by MDA only for the colony area detected in the first phase. Spectra extracted from each colony area were used as the training data for MDA. In the results of identification, there were isolated pixels like pepper noises which were not reasonable as real bacteria. To reduce such noises, $5 \times 5$ majority filter was applied. Final results of identification were pseudo-colored as shown in Section 3. Evaluation of detection and identification for colonies with various sizes was carried out in Subsection 3.3. Evaluation method is shown in Subsection 2.4. 


\subsection{Evaluation}

Evaluation of detection and identification was done by comparing with known results. First, mean absorption image was made. Colonies and background area were manually selected from the mean absorption image. We call them as known results and used for evaluation. The known result of largest colony in each species was used for training data in MDA for identification described in Subsection 2.3. Uncertain area was not used.

Figure 4 shows the schematic diagram of (a) ideal and (b) six possible results for identification. In case bacteria A exists in dashed circle and no other bacteria exist in the Petri dish as shown in Figure 4(a), identified result in the circle has three possibilities as shown in Figure 4(b), i.e., $n_{11}, n_{12}$ and $n_{13} . n_{11}$ is correct answer, $n_{12}$ is bacteria at correct place but wrong species and $\mathrm{n}_{13}$ is wrong material (background) at wrong place. Identified result in the background area has also three possibilities, i.e., $\mathrm{n}_{21}, \mathrm{n}_{22}$ and $\mathrm{n}_{23} . \mathrm{n}_{21}$ is correct bacteria at wrong place, $\mathrm{n}_{22}$ is wrong bacteria at wrong place and $\mathrm{n}_{23}$ is correct answer. Using these values, correct detection ratio and correct identification ratio were defined as shown in Eq. 1-6,

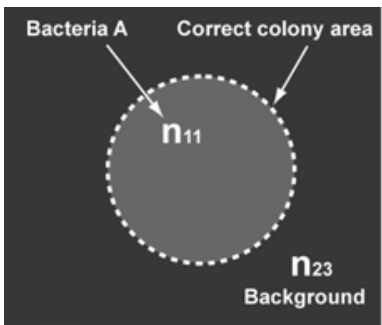

(a)

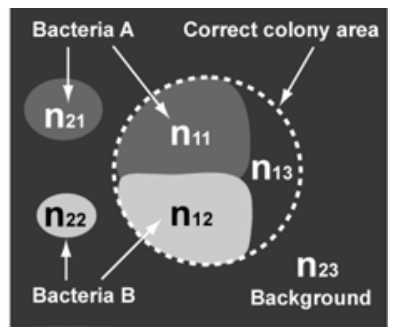

(b)

Fig. 4. Schematic diagram of (a) ideal and (b) six possible results for identification. In case bacteria A exists in dashed circle as shown in (a), identified result in the circle has three possibilities as shown in (b), i.e., $\mathrm{n}_{11}, \mathrm{n}_{12}$ and $\mathrm{n}_{13}$. Identified result in the background area has also three possibilities, i.e., $\mathrm{n}_{21}, \mathrm{n}_{22}$ and $\mathrm{n}_{23}$. Using these values, correct detection and identification ratio were defined

$$
\begin{gathered}
\mathrm{D}_{\mathrm{mc}}=\left(\mathrm{n}_{11}+\mathrm{n}_{12}\right) /\left(\mathrm{n}_{11}+\mathrm{n}_{12}+\mathrm{n}_{13}\right) . \\
\mathrm{D}_{\mathrm{bk}}=\mathrm{n}_{23} /\left(\mathrm{n}_{21}+\mathrm{n}_{22}+\mathrm{n}_{23}\right) \\
\mathrm{D}=\left(\mathrm{D}_{\mathrm{mc}}+\mathrm{D}_{\mathrm{bk}}\right) / 2 . \\
\mathrm{C}_{\mathrm{mc}}=\mathrm{n}_{11} /\left(\mathrm{n}_{11}+\mathrm{n}_{12}+\mathrm{n}_{13}\right) . \\
\mathrm{C}_{\mathrm{bk}}=\mathrm{n}_{23} /\left(\mathrm{n}_{21}+\mathrm{n}_{22}+\mathrm{n}_{23}\right) \\
\mathrm{C}=\left(\mathrm{C}_{\mathrm{mc}}+\mathrm{C}_{\mathrm{bk}}\right) / 2 .
\end{gathered}
$$


where $\mathrm{D}_{\mathrm{mc}}, \mathrm{D}_{\mathrm{bk}}$ and $\mathrm{D}$ are correct detection ratio of bacteria, background and in an image which is the average of $\mathrm{D}_{\mathrm{mc}}$ and $\mathrm{D}_{\mathrm{bk}}$, respectively. $\mathrm{C}_{\mathrm{mc}}, \mathrm{C}_{\mathrm{bk}}$ and $\mathrm{C}$ are correct identification ratio of bacteria, background and in an image which is the average of $\mathrm{C}_{\mathrm{mc}}$ and $\mathrm{C}_{\mathrm{bk}}$, respectively. In detection, species of bacteria were not considered.

\section{Results}

Detection and identification results for relatively large colonies shown in Figure 1(A) and microcolonies shown in Figure 1(B) were described in Subsection 3.1 and 3.2, respectively.

\subsection{Large Colonies}

Figure 5(A) shows mean absorption images, diameters of colonies and known results with numbers of groups. The size of the image is $240 \times 201$ pixels which correspond to about $2232 \times 1869 \mu \mathrm{m}(9.3 \mu \mathrm{m} /$ pixel $)$. All colonies in Figure 5(A) are the largest ones in each species taken with 4-power object lens. Numbers of groups were assigned as 1: Background, 2: Paracoccus pantotrophus, 3: Rhodopseudomonas palustris, 4: Rhodobium marinum and 5: Rhodobacter veldkampii. Figure 6(A) shows

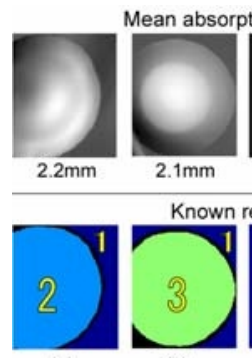

(a) (b)

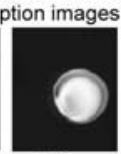

$1.0 \mathrm{~mm}$

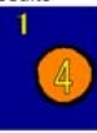

(c)

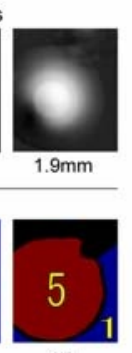

(d)

(A)

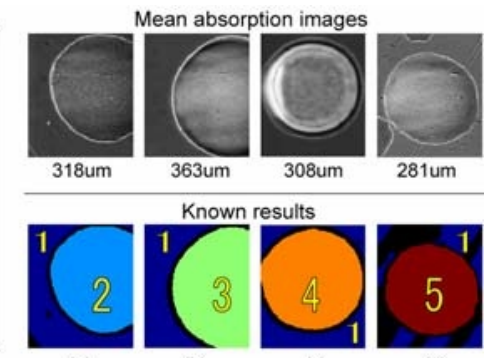

(b) (c)

(d)

Fig. 5. Mean absorption images, diameters of colonies and known results with numbers of groups. (A) Large colonies, (B) microcolonies

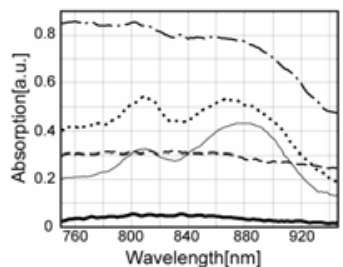

(A)

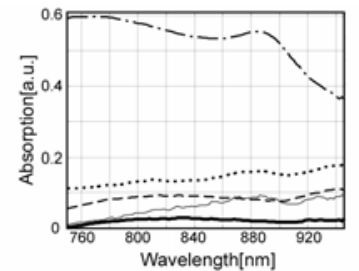

(B)

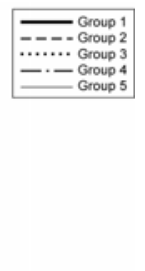

Fig. 6. Examples of absorption spectra averaged in each group. (A) Large colonies, (B) microcolonies 
examples of absorption spectra averaged in each group. Absorption peaks caused by pigment-protein complexes with Bchl $a$ were seen even though the spectral shape was smooth compared with Figure 2. The reason of the smoothness was that colonies were not chemically preprocessed at all and pigment-protein complexes with Bchl $a$ was covered with other materials such as cell membrane. Absorption spectra at different pixels are also shown in Figure 7. Absorption at the center of a colony is large, but still dull shaped because of the cell membrane.
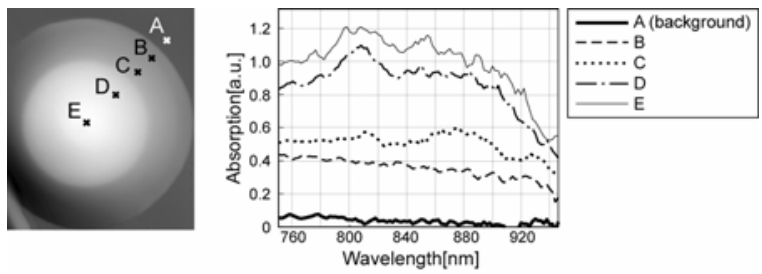

Fig. 7. Absorption spectra at different pixels. Absorption at the center of a colony is large, but still dull shaped because of the cell membrane

Figure 8(A) shows, from left to right, mean absorption images, known results and identification results for each species. Correct detection ratio D and correct identification ratio $\mathrm{C}$ are, (a) $94.8 \%, 92.0 \%$, (b) $96.0 \%, 96.0 \%$, (c) $99.8 \%, 96.8 \%$ and (d) $85.5 \%, 85.5 \%$, respectively. Most species were correctly identified.

(A)

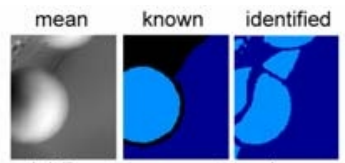

(a) Paracoccus pantotrophus

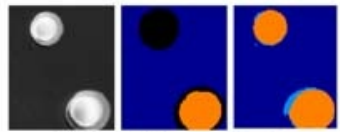

(c) Rhodobium marinum

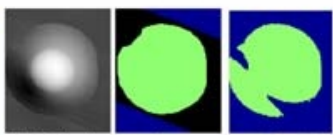

(b) Rhodopseudomonas palustris

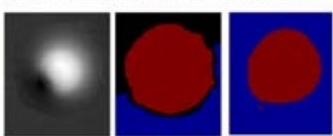

(d) Rhodobacter veldkampii

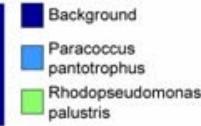

Rhodobium

Rhodobacter

veldkampii
(B)

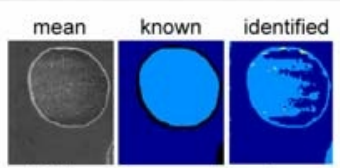

(a) Paracoccus pantotrophus

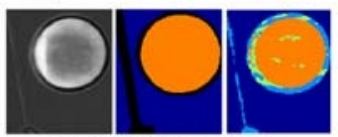

(c) Rhodobium marinum

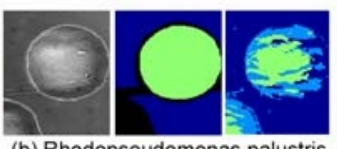

(b) Rhodopseudomonas palustris

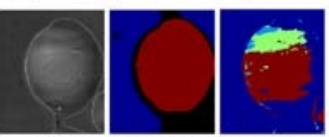

(d) Rhodobacter veldkampii

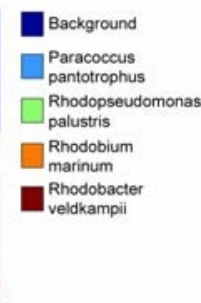

Fig. 8. Mean absorption image, known results and identification results. (A) Large colonies, (B) microcolonies 


\subsection{Microcolonies}

Figure 5(B) shows mean absorption images, diameters of colonies and known results with numbers of groups. The size of the image is $240 \times 201$ pixels which correspond to about $360 \times 101 \mu \mathrm{m}(1.5 \mu \mathrm{m} /$ pixel $)$. All colonies in Figure 5(B) are the largest ones in each species taken with 10-power object lens. Numbers of groups were assigned as 1: Background, 2: Paracoccus pantotrophus, 3: Rhodopseudomonas palustris, 4: Rhodobium marinum and 5: Rhodobacter veldkampii. Figure 6(B) shows examples of absorption spectra averaged in each group. Compared with Figure 6(A), absorption peaks caused by pigment-protein complexes with Bchl $a$ could not be seen clearly, because thicknesses of colonies were quite thin and absorption became low.

Figure 8(B) shows, from left to right, mean absorption images, known results and identification results for each species. Correct detection ratio $\mathrm{D}$ and correct identification ratio $\mathrm{C}$ are, (a) $80.6 \%, 80.1 \%$, (b) $88.5 \%, 70.8 \%$, (c) $99.9 \%, 88.9 \%$ and (d) $84.6 \%, 75.7 \%$, respectively. Compared with Figure 8(A), correct ratio was low. The reason of the error was mis-identification of bacteria (b), (c), (d) to background or bacteria (a). This is understandable, because background and bacteria (a) do not have any absorption peaks in the wavelength region and it is easy to confuse with those.

\subsection{Limit of Colony Size}

Evaluation of detection and identification of colonies with various sizes was carried out. Figure 9 shows the relation between colony size and (A) correct detection D, (B) correct identification $\mathrm{C}$, respectively. The number of colonies were 5(Group2), 3(Group3), 4(Group4), 5(Group5) and total 17 in Figure 9(A) and 9(Group2), 10(Group3), 10(Group4) and 10(Group5) and total 39 in Figure 9(B). In Figure 9(A) and (B), the colonies of these bacteria used in the experiment were correctly detected / identified with the ratio of over $60 / 80 \%$ in case the colony size was over $100 / 300$ $\mu \mathrm{m}$, respectively. In this experiment, background was almost $100 \%$ correctly detected and identified. It means that the error came from colony area. For example, correct detection $\mathrm{D}=60 \%$ means not 60 colonies in 100 colonies can be detected, but background area of $100 \%$ and colony area of $20 \%$ can be detected. In the viewpoint of microbiology, $60 \%$ for detection is accurate enough because colony area of $20 \%$ can

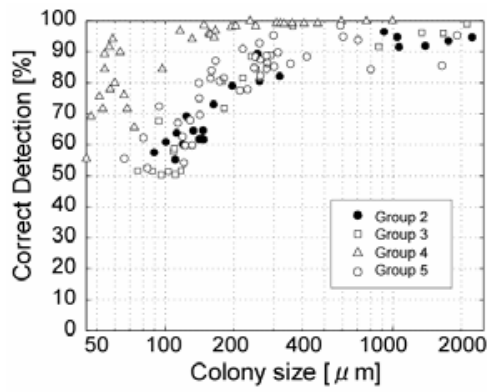

(A)

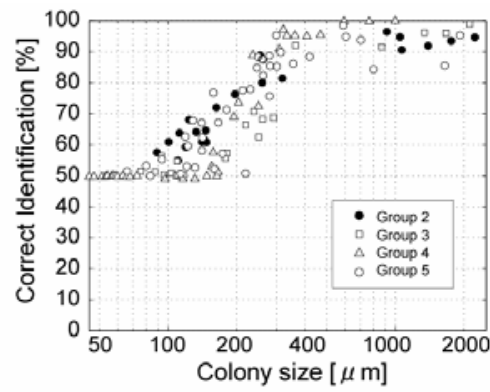

(B)

Fig. 9. The relation between (A) colony size and correct detection, (B) correct identification 
be easily counted as a single colony. $100 \%$ detection ratio is not necessary. Therefore, the limit of colony size for detection and identification with sufficient accuracy is about $100 \mu \mathrm{m}$ and $300 \mu \mathrm{m}$ from the microbiological viewpoint, respectively.

\section{Discussion and Conclusions}

We presented an application of the spectral imaging technique to detect and identify microorganisms for rapid food inspection. Colonies having a diameter of about 100 $\mu \mathrm{m}$ and $300 \mu \mathrm{m}$ were detected and identified with sufficient accuracy for every species used in this study, respectively. This means that the detection time can be shorten less than a half and the identification time can be shorten about several weeks compared with the conventional methods such as spectrophotometer which needs weeks for sample preparation.

The main advantages of this technique are the following: living microcolonies can be detected and identified without any chemical pretreatment such as staining, a single colony on the surface of Petri dish can be directly used as a sample, no extended culturing is necessary to prepare samples, alien substances cannot be mis-detected since spectral information is used.

In this study, we used photosynthetic bacteria as model bacteria to evaluate the validity of this method. Next steps in this research are as follows: to detect and identify contaminated samples with several species in a Petri dish, to fix target bacteria and to customize culturing and measuring condition for rapid food inspection at food processing plant. In practice, selection of appropriate culture media and/or selection of wavelengths can be considered. At this stage, spectral resolution was $2 \mathrm{~nm}$ and 99 images were measured for one spectral image. Precise spectral information, however, is not needed if a few spectral channels were well chosen. Dedicated spectral imaging system with a few filters could be designed in the future.

\section{Acknowledgements}

We appreciate microbial samples provided by The Department of Ecological Engineering, Toyohashi University of Technology, Dr. Hiroyuki Futamata, Ms. Yoko Okubo, and others that have assisted in this study. This work was partially supported by Cooperation of Innovative Technology and Advanced Research in Evolutional Area.

\section{References}

1. M. W. Griffiths, Rapid microbiological methods with hazard analysis critical control point, J AOAC Int. 1997 Nov-Dec;80(6):1143-50.

2. T. C. George, D. A. Basiji, B. E. Hall, D. H. Lynch, W. E. Ortyn, D. J. Perry, M. J. Seo, C. A. Zimmerman and P. J. Morrissey, Distinguishing modes of cell death using the ImageStream multispectral imaging flow cytometer, Cytometry Part A, 59A, 237-245, 2004.

3. Z. Fu, S. Rogelj and T. L. Kieft, Rapid detection of Escherichia coli O157:H7 by immunomagnetic separation and real-time PCR, Int J Food Microbiol. 2005 Mar 1;99(1):47-57. 
4. M. Sugiura, M. Takano, S. Kawakami, K. Toda and S. Hanada, Application of a Portable Spectrophotometer to Microbial Mat Studies, Microbes and Environments, vol.16, No.4, 255-261, 2001.

5. T. Zimmerman, J. Pietodorf, R. Pepperkok, Spectral imaging and its applications in live cell microscopy, FEBS Letters 546, 87-92, 2003.

6. M. Sunamura, A. Maruyama, T. Tsuji and R. Kurane, Spectral imaging detection and counting of microbial cells in marine sediment, J. of Microbiological methods, 53 57-65, 2003.

7. A. P. Arkin, E. R. Goldman, S. J. Robes, C. A. Goddard, W. J. Coleman, M. M. Yang and D. C. Youvan, Applications of imaging spectroscopy in molecular biology II. Colony screening based on absorption spectra, Bio/Technology, vol.8, 746-749, August, 1990.

8. D. C. Youvan, Imaging sequence space, Nature, vol.369, 79-80, 5 May 1994. 